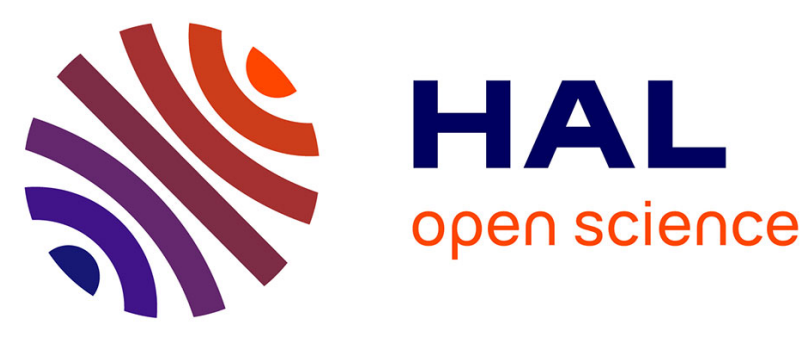

\title{
Effect of a collector bag for measurement of postpartum blood loss after vaginal delivery: cluster randomised trial in 13 European countries.
}

Wei-Hong Zhang, Catherine Deneux-Tharaux, Peter Brocklehurst, Edmund Juszczak, Matthew Joslin, Sophie Alexander, Euphrates Group

\section{To cite this version:}

Wei-Hong Zhang, Catherine Deneux-Tharaux, Peter Brocklehurst, Edmund Juszczak, Matthew Joslin, et al.. Effect of a collector bag for measurement of postpartum blood loss after vaginal delivery: cluster randomised trial in 13 European countries.. BMJ / BMJ (CLINICAL RESEARCH ED); Br Med J; British Medical Journal; Brit Med J, 2010, 340 (c293), pp.c293. inserm-00455479

\section{HAL Id: inserm-00455479 https://www.hal.inserm.fr/inserm-00455479}

Submitted on 10 Feb 2010

HAL is a multi-disciplinary open access archive for the deposit and dissemination of scientific research documents, whether they are published or not. The documents may come from teaching and research institutions in France or abroad, or from public or private research centers.
L'archive ouverte pluridisciplinaire HAL, est destinée au dépôt et à la diffusion de documents scientifiques de niveau recherche, publiés ou non, émanant des établissements d'enseignement et de recherche français ou étrangers, des laboratoires publics ou privés. 
1 Effect of a collector bag for measurement of postpartum blood loss after vaginal

delivery: a cluster randomised trial in thirteen European countries

Authors

Wei-Hong Zhang (Senior researcher) ${ }^{*}$, Catherine Deneux-Tharaux (Senior researcher) $\dagger$, Peter

Brocklehurst (Professor of Perinatal Epidemiology)

Statistician) $\$$, Matthew Joslin (General Practitioner) ${ }^{*}$, Sophie Alexander (Professor of Public

Health) $^{*}$ and the EUPHRATES Group ${ }^{\S}$

*Perinatal Epidemiology Research Unit, School of Public Health, Université Libre de Bruxelles (ULB), Belgium.

$\dagger$ INSERM, UMR S953, UPMC, Epidemiological research unit on perinatal health and women's and children's health. Bâtiment de recherche Hôpital Tenon 4, rue de la Chine 75020 Paris, France.

\$National Perinatal Epidemiology Unit, University of Oxford. Old Road Campus, Headington,

Oxford OX3 7LF, United Kingdom.

$\S$ EUPHRATES (EUropean Project on obstetric Haemorrhage, Reduction, Attitudes, Trial and Early warning System) collaborators : Sophie Alexander (Project leader, Belgium), Diogo Ayres-de-Campos (Portugal), Istvan Berbik (Hungary), Marie-Hélène Bouvier-Colle (France), Gérard Bréart (France), Peter Brocklehurst (United Kingdom), Vicenç Cararach (Spain), Anna Maria Marconi (Italy), Catherine Deneux-Tharaux (France), Risto Erkkola

21 (Finland), Mathias Klein (Austria), Jens Langhoff-Roos (Denmark), Alison Macfarlane (United Kingdom), Walter Prendiville (Ireland), Jos van Roosmalen (Nederlands), Babill

Wei-Hong Zhang (Belgium) 
1 Keywords: severe post-partum haemorrhage, collector bag, cluster-randomised controlled

2 trial, Europe

3 CORRESPONDING AUTHOR:

4 Wei-Hong ZHANG, M.D., MPH. PhD

5 Unité de recherche en Santé Reproductive et Epidémiologie Périnatale, Ecole de Santé

6 Publique, Université Libre de Bruxelles (ULB), Route de Lennik 808, CP 597, B-1070

7 Bruxelles, Belgium

$8 \quad$ Tel: ++ 32255563 68, Fax: ++ 3225554049

$9 \quad$ Email: wzhang@ulb.ac.be

10 


\section{Abstract}

2 Background- Postpartum haemorrhage (PPH) remains a leading cause of maternal morbidity

3 and mortality worldwide. Delay in diagnosis and care for PPH has been reported. The

4 inaccuracy of visual estimation of postpartum blood loss has been demonstrated.

5 Objectives- To evaluate the effectiveness of the systematic use of a transparent plastic

6 collector bag for measurement of postpartum blood loss after vaginal delivery in reducing the

$7 \quad$ incidence of severe $\mathrm{PPH}$

8 Design- A cluster randomised trial

$9 \quad$ Setting- Thirteen European countries

10 Participants-78 maternity units and 25381 women who had a vaginal delivery

11 Interventions- Maternity units were randomly assigned to systematically use a collector bag

12 (intervention group), or to continue to visually assess postpartum blood loss after vaginal

13 delivery (control group)

14 Main outcome measures- The primary outcome was the incidence of severe PPH in vaginal 15 deliveries, defined as a composite of one or more of the following events: blood transfusion, intravenous plasma expansion, arterial embolisation, surgical procedure, admission to intensive care unit, treatment with recombinant factor VII, or death.

Results- The incidence of severe PPH was 189 out of 11037 of vaginal deliveries (1.71\%) in the intervention group compared to 295 out of 14344 in the control group (2.06\%). The difference was not statistically significant either in individual level analysis (adjusted odds ratio $0.82 ; 95 \%$ CI 0.26 to 2.53 ) or in cluster level analysis (difference in weighted mean rate adjusted for baseline rate $0.16 \%$; $95 \%$ CI $-0.69 \%$ to $1.02 \%$ ).

23 Conclusion- The use of a collector bag after vaginal delivery did not reduce the rate of severe 24 PPH as compared to visual estimation of postpartum blood loss. 
1 Trial registration: International Standard Randomised Controlled Trial Number (ISRCTN)

266197422.

3 


\section{Introduction}

2 Worldwide, postpartum haemorrhage ( $\mathrm{PPH})$ remains one of the leading causes of maternal

3 mortality $^{1}$ and the main component of severe morbidity ${ }^{2-5}$, jeopardizing the woman's fertility,

4 exposing her to risks of transfusion and intensive care, and incurring costs. From reports in

5 developed countries, about one percent of deliveries are associated with severe $\mathrm{PPH}^{3-6}$.

6 Decreasing the prevalence of severe PPH remains challenging. This appears all the more

7 important given the recent increase in the incidence of PPH reported in several developed

8 countries $^{2,7,8}$. Individual risk factors have been described but they poorly predict the

9 occurrence of $\mathrm{PPH}^{9,10}$. Interest has focused on care-processes as they are potentially

10 amenable to change. Studies of maternal deaths show that most deaths due to PPH involve

11 delayed and substandard care in the diagnosis and management of haemorrhage ${ }^{11-13}$. Similar

12 findings were drawn from a population-based study of severe non-lethal $\mathrm{PPH}^{14}$.

13 Delay in diagnosis and treatment of PPH may result from an underestimation of blood loss at

14 delivery. Assessment of post-partum blood loss, particularly following vaginal birth, is

15 recognised as difficult. Many studies demonstrate that visual estimates of peripartum blood

16 loss are frequently inaccurate ${ }^{15-21}$, showing an overestimation of blood loss at low volumes

17 and an underestimation at larger volumes, the magnitude of underestimation typically

18 increasing with the volume of haemorrhage.

19 The hypothesis of this study was that if blood loss is monitored and objectively measured by

20 collection in a transparent plastic bag, rather than being visually assessed, care-giver response

21 will be triggered more rapidly when excessive blood loss occurs. Specifically when bleeding

22 is excessive but before haemorrhage has become catastrophic, appropriate management will

23 take place without delay, so reducing the incidence of severe PPH. A preliminary study shows

24 that a plastic collector bag constitutes a simple instrument to diagnose haemorrhage in the

25 delivery room ${ }^{22}$. However, the impact of its use on PPH-related health outcomes has never 
1 been tested. Despite lacking evidence, the bag is routinely used in a significant proportion of

2 maternity units in Belgium, France, Italy, and Portugal (Euphrates survey ${ }^{23}$, unpublished data).

3 The objective of this trial was to evaluate the effectiveness of the systematic use of a

4 transparent plastic collector bag for measurement of postpartum blood loss after vaginal

5 delivery in reducing the incidence of severe $\mathrm{PPH}$.

6

\section{Methods}

\section{Trial design}

9 A cluster-randomised design with maternity unit was the unit of randomization. Given the

10 logistics of clinical practice on the delivery suite, contamination appeared to be inevitable in

11 an individual-patient randomised trial setting.

$12 \quad$ Setting

13 The sites selected for the trial comprised 78 maternity units in 13 European countries (see

14 Table1).

15 Participants

16 Maternity units

17 Maternity units were eligible if they had more than 200 vaginal deliveries annually (excluding

18 water births), and no previous policy of routine use of collector bags. In addition, to ensure

19 that the standard of care for management of the third stage of labour was similar across all

20 participating units, they had to comply with the EUPHRATES consensus statement on the

21 prevention and management of $\mathrm{PPH}^{24}$; a minimum standard, not a detailed guideline.

\section{Women}

23 In all maternity units of participating countries (except Denmark), all women undergoing a

24 vaginal delivery during the study period were included. In Denmark, enrolment into the study 
1 in each maternity unit was midwife-dependant; if a midwife agreed to participate, all his/her

2 vaginal deliveries were included.

\section{$4 \quad$ Randomization}

5 The random allocation was produced centrally by the National Perinatal Epidemiology Unit in

6 Oxford, UK. A stratified design was used to ensure that the two arms of the trial were as

7 similar as possible at baseline with respect to the stratification factors (i) country and (ii) size

8 of maternity unit (median split within country).

9 Maternity units were randomly allocated to either systematically use a collector bag after 10 vaginal delivery (intervention arm), or not use the bag (control group).

\section{Intervention}

The trial was implemented between January 2006 and May 2007, depending on the country. Prior to participation, each centre was visited by the national coordinator. At the visit, staff were reminded of the EUPHRATES consensus statement on the prevention and management of PPH and familiarised with the processes and the data collection instrument.

In the intervention group, a second visit from the national coordinator took place after randomisation, during which, use of the collector bag was explained to birth attendants with standard written instructions and a training video aid. The bag was to be placed under the pelvis of the mother as soon as the baby was born and before delivery of the placenta. It was transparent and graduated, allowing continuous monitoring of blood loss. It did not require sterilization and could be used in dorsal, lateral or lithotomy positions. Women delivering standing or crouching could be offered the opportunity to lie down for the third stage,

24 allowing the bag to be placed under their pelvis. The bag was to be left under the woman's buttocks until the birth attendant was no longer concerned about blood loss e.g. when the 
1 sanitary towel was applied to the vulva. Bags were purchased centrally and provided to each

2 cluster in the intervention arm.

3 In the control group, no collector bag was used, postpartum blood loss being visually assessed.

4 During the study period, use of collector devices was monitored to assess compliance with 5 allocation.

\section{Outcomes}

8 The primary outcome for the trial was the incidence of severe PPH following vaginal

9 deliveries, defined as a composite of all women who experienced one or more of the

10 following: blood transfusion, intravenous plasma expansion, arterial embolisation, surgical

11 procedure, admission to intensive care unit, treatment with recombinant factor VII and death.

12 Secondary outcomes were each of the components of the primary outcome, manual removal of the placenta and administration of prostaglandins after delivery.

\section{Data collection}

16 Each participating centre was asked to collect data from all women undergoing a vaginal delivery for a period of 4 months.

Data were collected during two time intervals: a 1-month period pre-randomisation (baseline period), and a 3-month period beginning immediately following randomisation in the control group (trial period). In the intervention group, the 3-month period of data collection followed

21 a 2-week training period during which the unit started using the collector bag on women

22 undergoing vaginal delivery.

23 Data were collected using a form filled in by the birth attendants for each vaginal delivery,

24 and included information on the woman's age, induction of labour, mode of delivery, number

25 of babies and birth weight, prophylactic uterotonics, and outcome data. Additionally, a second 
1 form was used for deliveries where severe PPH occurred, collecting detailed information

2 regarding delivery and $\mathrm{PPH}$ management. This form was used to cross-check criteria for the 3 primary outcome.

6 Sample size calculation took into account the cluster-randomised design; the intracluster

7 correlation coefficient was estimated to be 0.01 . Assuming an event rate for the primary outcome of $2.5 \%$ in the control group, in order to detect a decrease in the event rate to $1.5 \%$ (a $40 \%$ relative risk reduction) with $80 \%$ power, a 2 -sided significance level of $5 \%$ and an average cluster size of 300 women, 82 clusters (41 in each arm of the trial) were required ${ }^{25}$.

\section{Statistical analysis}

Participants/maternity units were analysed in the groups to which they were assigned regardless of the management received by individual women or deviation from the protocol. Baseline characteristics of maternity units and individual women were summarized with counts (percentages) for categorical variables, mean (standard deviation [SD]) for normally distributed continuous variables, or median (interquartile [IQR]) for other continuous variables. Comparative statistical analysis was performed at both individual and cluster level and took into account the effect of clustering. All statistical tests were two-sided (5\% significance level) and not adjusted for multiple comparisons. Statistical analyses were

21 performed using SPSS version 17 (SPSS) and Stata v10.0 software (Stata Corporation,

22 College Station, Texas, USA).

Individual woman level analysis - primary and secondary outcomes were compared between

24 the two study groups both unadjusted and adjusted for the effect of clustering. In order to 25 determine the magnitude and direction of any differences in outcomes between the two 
1 groups, crude odds ratios and 95\% confidence intervals were calculated. Furthermore, logistic

2 regression was used to adjust for clustering and key prognostic factors. The cluster

3 randomised design imparts a data structure that facilitates the calculation of a valid

4 intracluster correlation coefficient, $\rho$.

5 Cluster level analysis was only performed on the primary outcome. Some hospitals

6 contributed fewer events than others, and some recruited fewer women. We allowed these

7 hospitals to have less effect on the treatment estimate by weighting the analysis based on the

8 precision, i.e. calculating the weighted mean difference for the treatment comparison. A

9 weighted linear regression model was used to test the effect of the intervention on the rate of

10 severe PPH during the trial period, adjusting for the baseline rate, expressed as the weighted

11 mean difference (plus 95\% confidence interval).

\section{Ethical aspects}

14 Ethics approval was obtained in each country from relevant local or national research ethics committees. Consent to participate was taken from the maternity units. Because the procedure being tested was not invasive or different from current clinical practice, and because outcome data were routinely collected at maternity units and anonymously transmitted, no individual consent was sought.

\section{Role of the funding source}

21 The project was funded by the European Union (EU) under Framework 5 (contract QLG4-

22 CT-2001-01352). EU had no role in the design, management, data collection, analyses, or 23 interpretation of the data. EU had no role in the writing of the manuscript or in the decision to 24 submit for publication. 


\section{Results}

3 Figure 1 shows the flow of maternity units and women through the study. Of the 84 maternity

4 units meeting the inclusion criteria, two maternity units declined to participate before

5 allocation. Forty one maternity units were randomised to the intervention group and 41 to the

6 control group. Two maternity units in each group opted out before receiving notification of

7 allocation because they lacked the necessary resources. Thirty-nine maternity units in each

8 group completed the trial. Table 1 shows the number of participating maternity units and

9 women included in each country.

10 One maternity unit did not collect baseline data in the intervention group. Deviating from the 11 protocol, the majority of maternity units (31 of 39) continued collecting data during the 2week training period in the intervention arm. In these units, trial data collection started after

13 the first month of baseline data collection. Four units in the control group collected trial data

14 for more than 3 months (up to 5 months). Only the 3-month period of data collection specified

15 in the protocol was considered for all units. In some Austrian hospitals, the number of women included was low, given the total expected number of deliveries. The national coordinator confirmed that the missing data were all caesarean deliveries, and that in some hospitals the caesarean rate was very high. Nevertheless, sensitivity analyses were performed, and showed that excluding these hospitals or even the entire Austrian data set did not influence the results.

\section{Characteristics of maternity units and women}

Baseline data were collected for 4937 in the intervention group and 4758 vaginal deliveries in

23 the control group and characteristics of maternity units and women (Table 2) were broadly

24 similar in the two groups for all factors, except for manual removal of the placenta and

25 prophylactic uterotonics, which were more common among women in the intervention group. 


\section{$1 \quad$ Primary outcome}

$2 \quad$ Individual level analysis

3 A total of 25381 women were included in the analysis (11037 in the intervention group and

414344 in the control group). The greater number of women in the control group was due to a

5 larger median cluster size (241 and 284 in the intervention and control groups, respectively)..

6 The incidence of severe PPH was 189 out of 11037 of vaginal deliveries (1.71\%) in the

7 intervention group compared to 295 out of 14344 in the control group (2.06\%). The difference

8 was not statistically significant (Table 3 ). The crude odds ratio for the effect of the

9 intervention was 0.83 (95\% CI, 0.69 to 1.00 ). The odds ratio adjusted for clustering was 0.83

10 (95\% CI, 0.27 to 2.60); after further adjustment for age, prophylactic uterotonics in the third

11 stage, mode of delivery and birth weight, the odds ratio was 0.82 (95\% CI, 0.26 to 2.53 ).

12 Sensitivity analyses were conducted to test the robustness of this result excluding units deviating from the protocol, and also by country, and by baseline rate of severe PPH (median split by country); these analyses provided similar results.

Cluster level analysis

The weighted mean severe PPH rate was $1.71 \%$ (SD 2.51) in the intervention group and $2.06 \%$ (SD 3.52) in the control group. The intracluster correlation coefficient for severe PPH was 0.023 . There was no significant difference in the rate of severe PPH between the two groups (weighted mean difference $-0.34 \%,(-2.56 \%$ to $1.87 \%)$; $=0.75)$. Adjusting for the baseline rate of severe PPH resulted in a slight change in this result (adjusted weighted mean difference $0.16 \%,(-0.69 \%$ to $1.02 \%) ; \mathrm{p}=0.70)$. Rates of severe PPH in the baseline and trial periods for each maternity unit were heterogeneous across units in different countries (Figure 2).

Figure 3 shows the difference in baseline and trial rates of severe PPH for each unit in the intervention group, according to the compliance of bag usage. There was no relationship 
1 between the difference in severe PPH rates (baseline and trial) and the actual proportion of

2 bag use. The analysis of the intervention effect on the primary outcome, including in the

3 intervention arm only maternity units where the bag was used in at least $50 \%$ of vaginal

4 deliveries, showed no significant difference between the two groups (individual level analysis

5 adjusting for cluster and individual characteristics; adjusted OR 0.59, 95\% CI (0.23-1.53)).

Secondary outcomes (individual level analysis)

Analyses were performed to test the effect of the intervention on the main components of the primary outcome (Table 3). The proportion of blood transfusion, surgical procedure or embolisation and of manual removal of placenta, did not substantially differ between the intervention and control groups, whether after adjusting for cluster or after further adjusting for other prognostic factors. There were no maternal deaths. The proportions of receipt of intravenous plasma expanders and of prostaglandins use were different between intervention and control groups, but the differences were not significant after adjusting for clustering effect.

\section{Discussion}

\section{$\underline{\text { Strengths and limitations of study }}$}

In this cluster randomised trial conducted on 25381 vaginal deliveries in 78 maternity units of

2013 European countries, the systematic use of a collector bag after vaginal delivery did not modify the rate of severe forms of postpartum haemorrhage. There was no evidence of heterogeneity, the results not differing according to country or size of hospital. This trial provides new results on an unexplored although controversial aspect of care in the third stage of labour. Although objective measurement has been shown to increase the accuracy of postpartum blood loss assessment compared to visual estimation ${ }^{15-21}$, the routine use of a collector 
1 bag is not associated with a significant decrease in severe PPH. This result constitutes an important

2 contribution to the on-going debate on strategies to improve the care of women with $\mathrm{PPH}$ and

3 decrease the incidence of severe cases.

4 Additionally, the cluster-randomised design, the large number of clusters and their diversity

5 provide good external validity to this trial.

6 There were small deviations from the protocol for data collection, but sensitivity analyses showed

7 that none of these changed the internal validity of the trial.

8 There was large heterogeneity of baseline rates for the severe event between units ( 0 to $13.4 \%)$. In

9 theory, such a variation should be an asset, and reflect a broad range of levels of risk in the

10 participating maternity units. However, because these differences were strongly related to the

11 country, there remains some concern regarding the criteria in use for the management of PPH in

12 different parts of Europe. Again sensitivity analysis showed that this aspect did not alter the 13 results.

14 There was some heterogeneity in baseline data between the intervention and control groups.

15 Heterogeneity in PPH-related practices and PPH rates has been reported across maternity

16 units in Europe, both between and within countries ${ }^{4,23}$. Although randomization is expected

17 to balance these differences between the two arms, the number of units randomized, although

18 large for a cluster RCT, makes residual imbalance possible although probably very slight.

19 However, analyses were adjusted for the main determinants of PPH (individual level analysis),

20 and baseline rate of severe PPH (cluster-level analysis); in addition, sensitivity analysis

21 indicated that the absence of significant impact of the intervention was similar whether the

22 maternity units had high or low baseline rate of severe PPH. In consequence, any perceived or 23 real imbalance in these characteristics should have little or no impact on the findings.

\section{Hypotheses for the results}


1 Different mechanisms may explain the absence of difference in the rates of severe PPH between

2 maternity units which used the bag and those where blood loss was visually assessed.

3 This may be due to a lack of compliance to the intervention. However, the persistent absence

4 of difference between the 2 groups when the analysis was restricted to the units where the bag

5 was used in a high proportion of deliveries suggests this is unlikely.

6 One potential reason for the apparent ineffectiveness of the intervention might be that the

7 bags were actually not used correctly; in particular, there might be concern that the bags were covered most of the time and thus could not be viewed. However, because detailed oral and written instructions were provided and the training video clearly showed the care giver watching the bag and the graduations, such misuse is unlikely to explain the observed lack of

11 effect.

12 Participation in the study may indicate a particular interest in the management of PPH so that existing management had little room for improvement. However, the variety of baseline rates

14 of severe PPH in these units makes such a selection process unlikely.

It may be hypothesized that the intervention has a double effect, in two opposite directions:

16 increasing the rate of ascertainment through increased vigilance and decreasing the prevalence rate

17 through timely management of excessive bleeding. If these two components were of the same order of magnitude, the global effect would be no effect. However, if this explanation was realistic, one would expect different size of effects with different baseline rates and/or different degrees of compliance. None of this occurred, making it unlikely that a benefit of the intervention

21 in terms of decreased severe outcome was balanced by an equivalent increase in ascertainment. In 22 fact the intervention appeared to increase PPH rates, reflecting possibly, that the intervention was 23 more effective on improving ascertainment than on changing practice.

24 A concomitant effect in the control group may also have contributed to the absence of 25 difference between the two arms. Contamination of the intervention to control units is 
1 unlikely since participating units were not in contact, and no use of bags was reported in any

2 control unit. Participation in a research study, independently of any specific intervention, has

3 been reported to change behaviors of participants (Hawthorne effect ${ }^{26}$ ). The hypothesis that

4 the management of PPH would have improved in the control arm is, however, not supported

5 by the absence of change in the rate of severe PPH between the baseline and trial periods in

6 this group.

7 The most plausible explanation of the negative result of this trial is that having a more

8 accurate assessment of postpartum blood loss is not, by itself, sufficient to change behaviors

9 of care givers and improve PPH management. Lack of identification of women with excessive

10 postpartum bleeding is a considerable problem, potentially leading to higher levels of medical

11 intervention if the bleeding progresses to severe haemorrhage. We designed a strategy to

12 increase care-givers awareness. The fact that this has not translated into a change in clinical

13 outcomes probably reflects the complexity of management decisions, which are influenced by

14 multiple factors such as organization of the delivery ward, and how care givers perceive and

15 cope with emergencies.

\section{Comparison with other studies}

17 We did not find any other published study assessing the effectiveness of the collector bag.

However we have identified other large multicentre randomised trials in the field of maternal and child health where a diagnostic or screening test was evaluated without any associated instructions about the management of abnormal results ${ }^{27-29}$. None of these trials showed

21 benefit with the introduction of the test. In addition Althabe et al have shown that simple 22 information is not sufficient to impact birth attendants readiness to change ${ }^{30}$. These various reports suggest that the effect of enhanced diagnostic methods should include an accompanying protocol of management, and maybe a specific behavioral intervention, which in effect becomes a "complex intervention". 


\section{$1 \quad$ Conclusions and policy implications}

2 The practical implication of these results for high income countries, is that those units which

3 are using a collector bag (at a cost between 1 and $11 €$ per bag in Europe) need to reconsider

4 their practice, and maybe reallocate the resources to other aspects of care. Units which are not

5 routinely using the bag should keep the same policy. For resource poor countries positive

6 results of the use of the "kanga collector" have been reported ${ }^{31}$. This needs to be tested in a

7 randomised design. In the current context of reported on-going increase in the prevalence of

$8 \mathrm{PPH}$, further research is needed to develop and test effective strategies to decrease the

9 prevalence of severe PPH through improvement of management. These will probably be

10 multifaceted interventions, and in this context, the collector bag may warrant further

11 investigation.

12 
1 « What this paper adds » box

\section{What is already known on this subject}

3 Delay in diagnosis and initial care for postpartum hemorrhage (PPH) has been reported, and

4 may result from an underestimation of postpartum blood loss, due to the inaccuracy of visual

5 assessment. A collector bag has been proposed as a useful tool to objectively measure

6 postpartum blood loss. However, the impact of its use has never been tested. Despite lacking

7 evidence, the bag is routinely used in a significant proportion of maternity units in Europe.

8

\section{What this study adds}

10 Our study suggests that, for western countries, the routine use of a collector bag to objectively

11 assess postpartum blood loss after vaginal delivery, without specific guideline regarding

12 threshold and action, does not reduce the incidence of severe PPH. 


\section{Acknowledgements}

2 The project was funded by the European Union (EU) under Framework 5 (contract QLG4-

3 CT-2001-01352).

4 We are grateful to Professors Allan Donner (Canada) and Pierre Buekens (USA) for their

5 scientific advice; to Stéphane Freze and Myriam Loubriat for their contribution to data

6 collection and cleaning.

7 The following people played an essential part in running the trial in their own country and units: Austria: Co-ordination -Sylvia Artner-Matuschek, Adolf Beck, Daniela Meger David;

KH Ried- Penzinger Monika; LKH Bad Ischl - Carola Fuschlberger-Traxler; LKH Heinz

Klagenfurt- Leipold; Hanusch KH- Daniela Meger David; AKH Univ- Hans Helmer,

Katharina Klein; LKH Kufstein- Andrea Ehm. Belgium: Ambroise Paré (Mons)- Gilles

Ceysens, Annick Nouls, Yaacoub Salame, Linda Van Lierde; Ath- Françoise Clerquin, Pierre

Delvoye, Jean Piret; UZ Gent- Paul Defoort, Marleen Temmerman; AZ VUB (Brussels)-

Maria Breugelmans, Lieve Devalckeneer, Monika Laubach; Baudour- Joël Annet, Renaud

Paquay, Valérie Vandenbosch; Brugmann (Brussels)- Thomas Pezin, Alain Vokaer; Erasme (Brussels)- Christine Kirkpatrick, Anne Maas; Hopital St Pierre (Brussels)- Patricia Barlow,

Julie Belhomme, Nordine Ben Ali, Daniel Murillo; Ieper- Colette Berten, Geert Page; Ixelles (Brussels)- Véronique Ziereisen; KUL (Leuven)- Bernadette Bijnens, Bernard Spitz, Joske Timmermans; St Jean (Brussels)- Xavier de Muylder, Christine Stoop; Ste Anne \& St Remi (Brussels)- Paul Befahy, Anne Fostier; Tivoli -Maria Fabbricattore , Jacques Francotte,

21 Sylvie Hollemaert; Tournai IMC- Viviane Gadenne; Vésale (Charleroi)- Patrick De Nayer,

22 Didier Oberweiss. Denmark: Co-ordination -Ane Rom, Birgitte Rode Diness; Gentofte 23 Hospital- Anne Barfoed; Glostrup- Ambika Ravn; Hillerød- Gitte Ulriksen; Slagelse- Karen 24 Marie Wigh Felsen; Holbæk- Marianne Brandstrup Larsen; Frederiksberg- Ane Rom. 
1 Finland: Helsinki University Central Hospital- Vedran Stefanovic; Midwifery Institute of

2 Helsinki- Veli-Matti Ulander; University central hospital of Turku- Risto Erkkola; University

3 Hospital of Tampere-Jukka Uotila. France: Moulin Hospital- Michel Beytout, Catherine

4 Damouret; University Hospital (Nancy)- Brigitte Guillemain; Antoine Béclère; University

5 Hospital (Clamart)-Aurélia Chauveaud; Tenon University Hospital (Paris)- Nadia Berkane,

6 Marie-Christine Chaux; University Hospital (Rouen)- Loic Marpeau, Sabine Sionville;

7 Villleneuve St Georges Hospital- Patricia Tran van. Hungary: Co-ordination - István Szabó;

8 Baranya County Hospital (Pécs)-József Bódis; City Hospital (Mosonmagyaróvár)- István

9 Barcza; Erzsébet Hospital (Sopron)- Károly Péter Csécsei; Petz Aladár Teaching Hospital

(Győr)- Sándor Gardó; Selye János Hospital (Komárom)- László Rokay; Szent Borbála

Hospital (Tatabánya)- Mihály Molnár; University Hospital (Sci. Univ. Pécs)- István Szabó,

Tamás Csermely; Vaszary Kolos Teaching Hospital (Esztergom)- István Berbik. Ireland:

Co-ordination -Reem Akkawi, Fidelma Cavanagh; Coombe Women's Hospital (Dublin)-

Suzanne Kelly; Our Lady of Lourdes (Drogheda)- Dalia Sikafi, Ann Keating; Cavan General

Hospital- Iram Basit, Marie McCusker; Midland Regional Hospital Mullingar- Mary Corbet.

Italy: Az. SS. Antonio e Biagio e C Arrigo- Enrico Rovetta; Osp di Bassano del Grappa-

Yoram Meir; Osp Civile San Paolo- Antonio Castellano; Osp Civile S Liberatore- Claudio Angeloni; Osp San Massimo di Penne- Quirino Di Nisio; Pres Osp Di Piove Di Sacco-

Antonino Oro. Netherlands: Co-ordination - Marlies Rijnders, Esteriek de Miranda; St Lucas

Hospital; Bronovo Hospital. Norway: Co-ordination -Bente Rønnes; Sykehuset Innlandet;

Gjovik- Anne Kari Gjestvang, Elham Mahjoob; Sykehuset Innlandet Elverum- Agneta

Stramrud. Portugal: Co-ordination - Maria Fatima Oliveira, Cristina Ferreirinha;

Maternidade Bissaya Barreto (Coimbra)- Ascenção Baía ; Maternidade Daniel de Matos

(Coimbra) - José Portugal; H. S. Marcos (Braga) - Lucília Guerra Sousa: H. S. Joao (Porto)-

Cristina Ferreirinha; Senhora da Oliveira (Guimaraes) - Alice Santos .Spain: Co-ordination - 
1 Sonia Pisa, Sara Herrero; H. Clínic- Enrique Barrau, Jordi Bellart, Isabel Salgado; H. Vall

2 d'Hebró- Anna Suy; H. Sabadell- Jordi Costa, Maria Grimau; H. Joan XXIII- Ramón Ma

3 Miralles; H. del Mar- Antoni Payà; H. San Joan de Deu- Sergi Cabré; H. Sant Pau- Marta

4 Simó; H. Germans Trias- José Lecumberri. Switzerland: Co-ordination -Irene Hösli, Gideon

5 Sartorius; Aarau-Monya Todesco; Basel- Gideon Sartorius; Frauenfeld- Verena Geissbühler;

6 Fribourg- David Stucki, Heidrun Schönberger; Solothurn- Suzanne Zakher; St Gallen- Gero

7 Drack, Anika Hey-Moonen. 
$1 \quad$ Figure 1

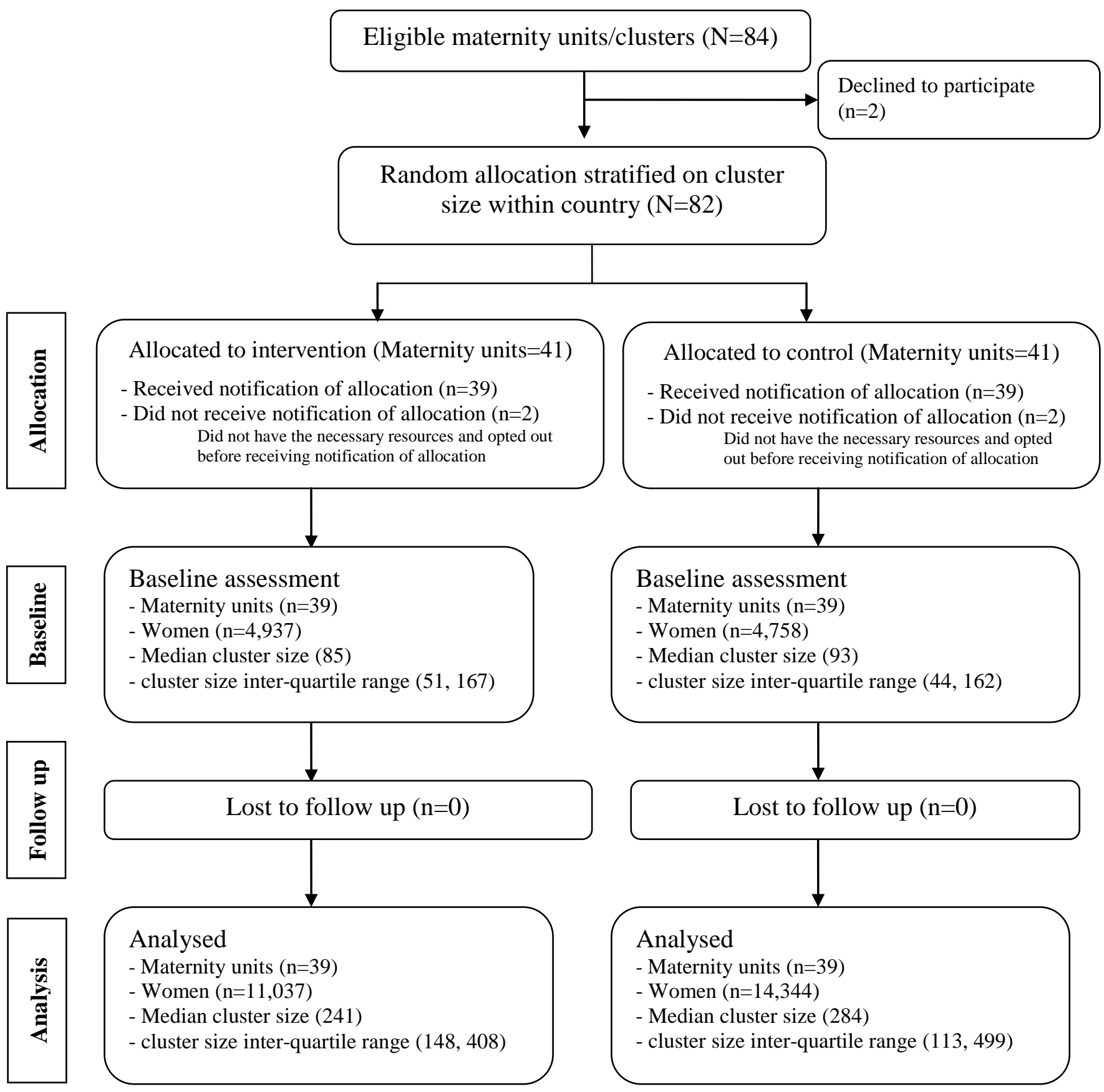


Table 1- Number of maternity units and women in baseline and trial periods by allocation and by country*

\begin{tabular}{|c|c|c|c|c|c|c|c|c|c|c|}
\hline \multirow{3}{*}{ Country } & \multicolumn{2}{|c|}{ Maternity units } & \multicolumn{8}{|c|}{ Women } \\
\hline & \multirow{2}{*}{$\begin{array}{c}\text { Intervention } \\
\mathbf{N}\end{array}$} & \multirow{2}{*}{$\begin{array}{c}\text { Control } \\
\mathbf{N}\end{array}$} & \multirow{2}{*}{$\begin{array}{r}\text { Total } \\
\mathbf{N}\end{array}$} & \multirow{2}{*}{$\begin{array}{r}\% \text { total } \\
(\%)\end{array}$} & \multicolumn{3}{|c|}{ Baseline period } & \multicolumn{3}{|c|}{ Trial period } \\
\hline & & & & & Total & Intervention & Control & Total & Intervention & Control \\
\hline Austria & 3 & 3 & 1067 & 3.0 & 371 & 219 & 152 & 696 & 359 & 337 \\
\hline Belgium & 8 & 8 & 6013 & 17.1 & 1552 & 728 & 824 & 4461 & 1867 & 2594 \\
\hline Denmark & 3 & 3 & 1657 & 4.7 & 507 & 272 & 235 & 1150 & 562 & 588 \\
\hline Finland & 2 & 2 & 4805 & 13.7 & 1347 & 656 & 691 & 3458 & 1551 & 1907 \\
\hline France & 3 & 3 & 3702 & 10.6 & 972 & 544 & 428 & 2730 & 1351 & 1379 \\
\hline Hungary & 4 & 4 & 2230 & 6.4 & 562 & 268 & 294 & 1668 & 784 & 884 \\
\hline Ireland & 2 & 2 & 3971 & 11.3 & 950 & 300 & 650 & 3021 & 946 & 2075 \\
\hline Italy & 3 & 3 & 926 & 2.6 & 196 & 138 & 58 & 730 & 491 & 239 \\
\hline Netherlands & 1 & 1 & 1084 & 3.1 & 301 & 130 & 171 & 783 & 322 & 461 \\
\hline Norway & 1 & 1 & 668 & 1.9 & 143 & 72 & 71 & 525 & 241 & 284 \\
\hline Portugal & 2 & 3 & 3274 & 9.3 & 810 & 338 & 472 & 2464 & 901 & 1563 \\
\hline Spain & 4 & 3 & 4351 & 12.4 & 1595 & 1097 & 498 & 2756 & 1239 & 1517 \\
\hline Switzerland & 3 & 3 & 1328 & 3.8 & 389 & 175 & 214 & 939 & 423 & 516 \\
\hline Total & 39 & 39 & 35076 & 100.0 & 9695 & 4937 & 4758 & 25381 & 11037 & 14344 \\
\hline
\end{tabular}

* Baseline data were unavailable in one maternity unit in the intervention group 
Table 2- Baseline characteristics of maternity units and individual women by allocation*

\begin{tabular}{|c|c|c|}
\hline & $\begin{array}{c}\text { Intervention } \\
\text { group }\end{array}$ & $\begin{array}{l}\text { Control } \\
\text { group }\end{array}$ \\
\hline Maternity units & $\mathrm{N}=38 \dagger$ & $\mathrm{N}=39$ \\
\hline \multicolumn{3}{|l|}{ Rate of caesarean delivery - (\%) } \\
\hline Median & 21.1 & 21.7 \\
\hline Interquartile range & $17.4-26.6$ & $14.6-26.0$ \\
\hline$>1600$ deliveries/yr - no. $(\%)$ & $20(52.6)$ & $19(48.7)$ \\
\hline Women & $\mathrm{N}=4937$ & $\mathrm{~N}=4758$ \\
\hline \multicolumn{3}{|l|}{ Age - yr } \\
\hline Mean & $29.6 \pm 5.4$ & $29.7 \pm 5.5$ \\
\hline Median & 30.0 & 30.0 \\
\hline Interquartile range & $26-33$ & 26- 33 \\
\hline Missing data - no. & 31 & 23 \\
\hline \multicolumn{3}{|l|}{ Mode of delivery - no. ( $\%)$} \\
\hline Spontaneous vaginal delivery & $4104(83.1)$ & $4062(85.4)$ \\
\hline Operative vaginal delivery & $833(16.9)$ & $696(14.6)$ \\
\hline Induction - no. (\%) & $1080(21.9)$ & $1043(21.9)$ \\
\hline \multicolumn{3}{|l|}{ Number of babies - no. $(\%)$} \\
\hline Single & $4833(98.5)$ & $4645(98.6)$ \\
\hline Multiple & $76(1.5)$ & $68(1.4)$ \\
\hline Missing data - no. & 28 & 45 \\
\hline \multicolumn{3}{|l|}{ Birth weight - grams } \\
\hline Mean & $3315 \pm 566.4$ & $3349 \pm 549.1$ \\
\hline Median & 3330 & 3370 \\
\hline Interquartile range & $3020-3660$ & $3050-3690$ \\
\hline Missing data - no. & 26 & 29 \\
\hline Prophylactic uterotonics in 3rd stage - no. (\%) & $3527(71.4)$ & $3153(66.3)$ \\
\hline Missing data - no. & 0 & 5 \\
\hline Prostaglandin used after birth - no. (\%) & $212(4.3)$ & $218(4.6)$ \\
\hline Missing data - no. & 0 & 5 \\
\hline Manual removal of the placenta - no. $(\%)$ & $204(4.1)$ & $121(2.5)$ \\
\hline Missing data - no. & 0 & 5 \\
\hline Severe PPH - no. $(\%)$ & $60(1.22)$ & $90(1.89)$ \\
\hline
\end{tabular}

* Plus-minus values are mean \pm SD. Severe PPH denotes severe Post-Partum Haemorrhage defined by one of the following: maternal death, transfusion, plasma expansion, surgery/embolisation, ICU, recombinant factor VII.

$\uparrow$ Baseline data were unavailable in one maternity unit. 
Table 3- Main outcomes*

\begin{tabular}{cccccc}
\hline Intervention & Control & ICC & Crude odds ratio (95\% & Adjusted OR & Adjusted OR \\
$\mathrm{N}=11037$ & $\mathrm{~N}=14344$ & $(\rho)$ & $\mathrm{CI})$ & $(95 \% \mathrm{CI}) \dagger$ & $(95 \% \mathrm{CI}) \dagger$ \\
no. $(\%)$ & no. $(\%)$ & & & &
\end{tabular}

\begin{tabular}{|c|c|c|c|c|c|c|}
\hline \multicolumn{7}{|l|}{ Primary outcome } \\
\hline \multirow[t]{2}{*}{ Severe PPH } & $189(1.71)$ & $295(2.06)$ & 0.023 & $0.83(0.69-1.00)$ & $0.83(0.27-2.60)$ & $0.82(0.26-2.53)$ \\
\hline & & & & $\mathrm{P}=0.05$ & $\mathrm{P}=0.8$ & $\mathrm{P}=0.7$ \\
\hline \multicolumn{7}{|l|}{ Secondary outcomes } \\
\hline \multirow[t]{2}{*}{ Blood transfusion } & $86(0.78)$ & $135(0.94)$ & 0.011 & $0.83(0.63-1.68)$ & $0.83(0.35-1.96)$ & $0.80(0.33-1.90)$ \\
\hline & & & & $\mathrm{P}=0.2$ & $\mathrm{P}=0.8$ & $\mathrm{P}=0.6$ \\
\hline \multirow[t]{2}{*}{ Plasma expander } & $127(1.15)$ & $222(1.55)$ & 0.022 & $0.74(0.59-0.92)$ & $0.74(0.20-2.72)$ & $0.95(0.62-1.46)$ \\
\hline & & & & $\mathrm{P}=0.007$ & $\mathrm{P}=0.7$ & $\mathrm{P}=1.0$ \\
\hline Surgical procedure or & $50(0.45)$ & $76(0.53)$ & 0.012 & $0.85(0.60-1.22)$ & $0.85(0.20-3.63)$ & $0.78(0.18-3.40)$ \\
\hline embolisation & & & & $\mathrm{P}=0.9$ & $\mathrm{P}=0.9$ & $\mathrm{P}=0.7$ \\
\hline Manual removal of & $326(2.95)$ & $366(2.55)$ & 0.016 & $1.16(1.00-1.35)$ & $1.16(0.76-1.77)$ & $1.09(0.72-1.67)$ \\
\hline placental & & & & $\mathrm{P}=0.05$ & $\mathrm{P}=0.5$ & $\mathrm{P}=0.7$ \\
\hline Prostaglandins use & $501(4.54)$ & $766(5.34)$ & 0.129 & $0.84(0.75-0.95)$ & $0.84(0.40-1.77)$ & $0.85(0.40-1.80)$ \\
\hline & & & & $\mathrm{P}=0.004$ & $\mathrm{P}=0.7$ & $\mathrm{P}=0.7$ \\
\hline
\end{tabular}

\footnotetext{
* Severe PPH denotes severe Post-Partum Haemorrhage defined by one of the following: maternal death, transfusion, plasma expansion, surgery/embolisation, ICU, recombinant factor VII. ICC denotes Intracluster Correlation Coefficient $(\rho)$

$\dagger$ Adjusted for clustering (maternity unit)

$\$$ Adjusted for clustering (maternity unit), age of mother, prophylactic uterotonics using in the third stage, mode of delivery and birth weight
} 
Figure 2

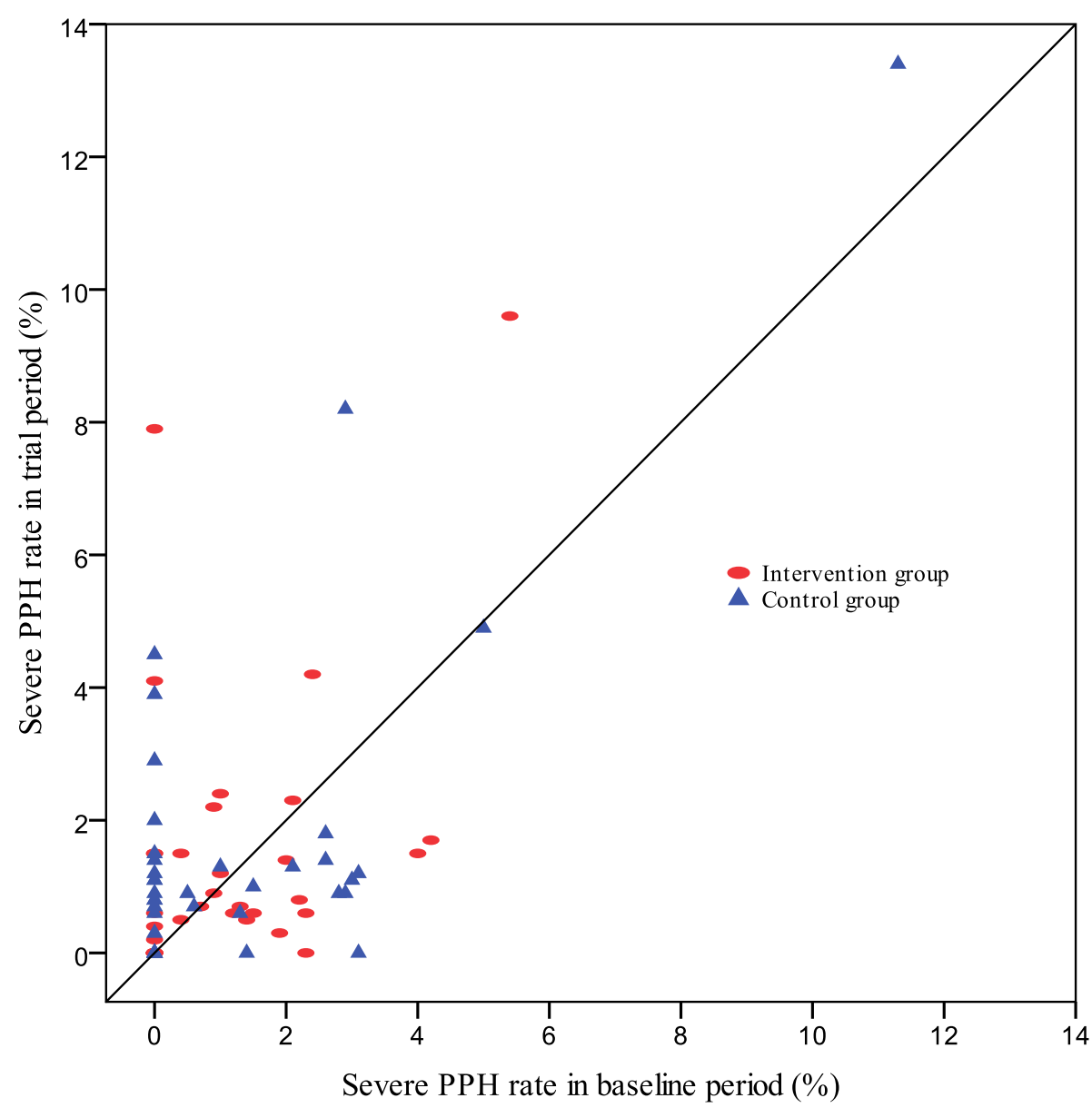


Figure 3

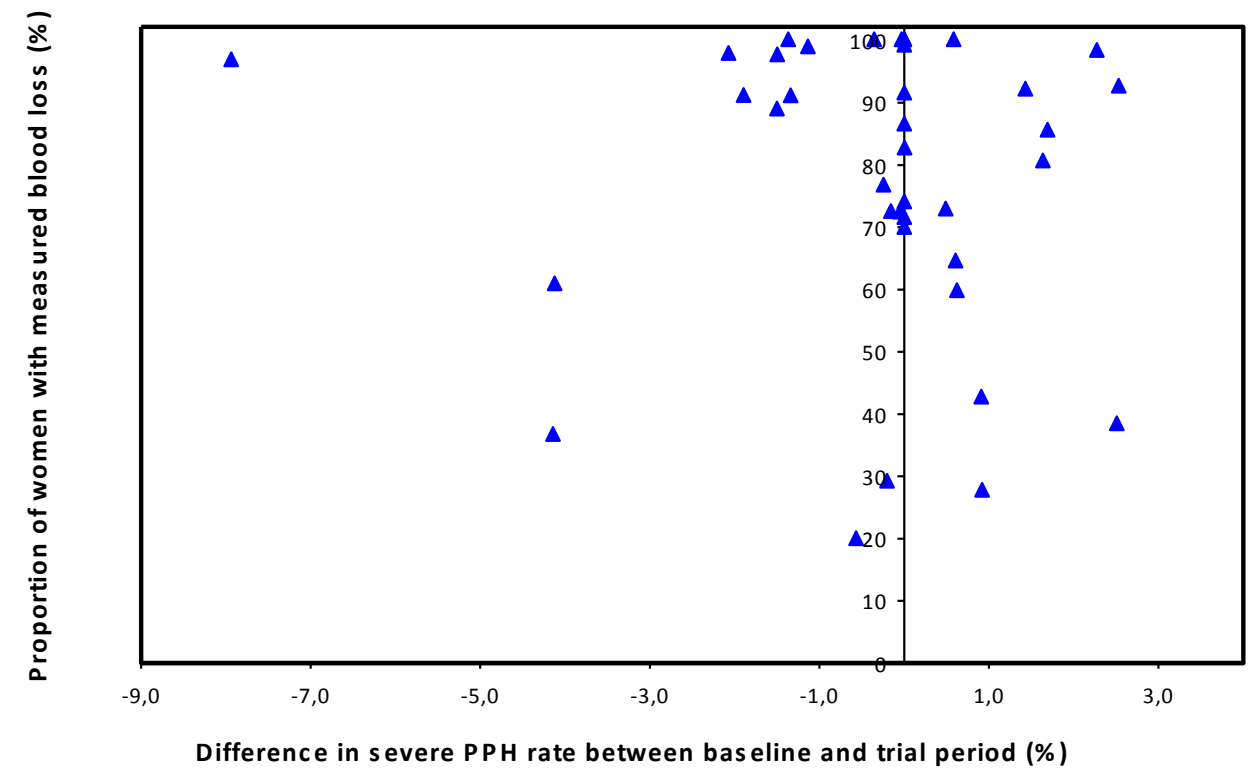




\section{Legends for figures}

Figure 1: Trial flow diagram

Figure 2: Rate of severe post-partum haemorrhage during baseline and trial periods for each maternity unit (Each dot represents one maternity unit. The diagonal line means no change in the PPH rate from baseline to trial period)

Figure 3: Difference in rate of severe post-partum haemorrhage (baseline rate- intervention rate) according to compliance with intervention (\% of women with measured blood loss) in the 38 units in the intervention group during the trial period 


\section{References}

1. Khan KS, Wojdyla D, Say L, Gulmezoglu AM, Van Look PF. WHO analysis of causes of maternal death: a systematic review. Lancet 2006;367:1066-74.

2. Callaghan WM, Mackay AP, Berg CJ. Identification of severe maternal morbidity during delivery hospitalizations, United States, 1991-2003. Am J Obstet Gynecol 2008;199:133 e1-8.

3. Wen SW, Huang L, Liston R, et al. Severe maternal morbidity in Canada, 1991-2001. Cmaj 2005;173:759-64.

4. Zhang WH, Alexander S, Bouvier-Colle MH, Macfarlane A. Incidence of severe preeclampsia, postpartum haemorrhage and sepsis as a surrogate marker for severe maternal morbidity in a European population-based study: the MOMS-B survey. Bjog 2005;112:89-96. 5. Zwart J, Richters J, Öry F, de Vries J, Bloermenkamp K, van Roosmalen J. Severe maternal morbidity during pregnancy, delivery and puerperium in the Netherlands: a natiowide populayion-based study of 371000 pregnancies. BJOG 2008;115:842-50.

6. Al-Zirqi I, Vangen S, Forsen L, Stray-Pedersen B. Prevalence and risk factors of severe obstetric haemorrhage. Bjog 2008;115:1265-72.

7. Joseph KS, Rouleau J, Kramer MS, Young DC, Liston RM, Baskett TF. Investigation of an increase in postpartum haemorrhage in Canada. Bjog 2007;114:751-9.

8. Roberts CL, Ford JB, Algert CS, Bell JC, Simpson JM, Morris JM. Trends in adverse maternal outcomes during childbirth: a population-based study of severe maternal morbidity. BMC Pregnancy Childbirth 2009;9:7.

9. Sherman SJ, Greenspoon JS, Nelson JM, Paul RH. Identifying the obstetric patient at high risk of multiple-unit blood transfusions. J Reprod Med 1992;37:649-52.

10. Mathai M, Gulmezoglu AM, Hill S. Saving womens lives: evidence-based recommendations for the prevention of postpartum haemorrhage. Bull World Health Organ 2007;85:322-3.

11. Rapport du Comité National d'Experts sur la Mortalité Maternelle (CNEMM). France 2006:http://www.invs.sante.fr/publications/2006/mortalite maternelle/rapport.pdf.

12. Berg CJ, Harper MA, Atkinson SM, et al. Preventability of pregnancy-related deaths: results of a state-wide review. Obstet Gynecol 2005;106:1228-34.

13. Lewis G. Saving mother's lives: reviewing maternal deaths to make motherhood safer2003-2005. the seventh report of the Confidential Enquiries into maternal deaths in the United Kingdom. London: CEMACH; 2007.

14. Bouvier-Colle MH, Ould El Joud D, Varnoux N, et al. Evaluation of the quality of care for severe obstetrical haemorrhage in three French regions. Bjog 2001;108:898-903.

15. Bose P, Regan F, Paterson-Brown S. Improving the accuracy of estimated blood loss at obstetric haemorrhage using clinical reconstructions. Bjog 2006;113:919-24.

16. Dildy GA, 3rd, Paine AR, George NC, Velasco C. Estimating blood loss: can teaching significantly improve visual estimation? Obstet Gynecol 2004;104:601-6.

17. Duthie SJ, Ven D, Yung GL, Guang DZ, Chan SY, Ma HK. Discrepancy between laboratory determination and visual estimation of blood loss during normal delivery. Eur J Obstet Gynecol Reprod Biol 1991;38:119-24.

18. Newton M, Mosey LM, Egli GE, Gifford WB, Hull CT. Blood loss during and immediately after delivery. Obstet Gynecol 1961;17:9-18.

19. Prasertcharoensuk W, Swadpanich U, Lumbiganon P. Accuracy of the blood loss estimation in the third stage of labor. Int J Gynaecol Obstet 2000;71:69-70.

20. Razvi K, Chua S, Arulkumaran S, Ratnam SS. A comparison between visual estimation and laboratory determination of blood loss during the third stage of labour. Aust $\mathrm{N}$ Z J Obstet Gynaecol 1996;36:152-4. 
21. Stafford I, Dildy GA, Clark SL, Belfort MA. Visually estimated and calculated blood loss in vaginal and cesarean delivery. Am J Obstet Gynecol 2008;199:519 e1-7.

22. Tourne G, Collet F, Lasnier P, Seffert P. [Usefulness of a collecting bag for the diagnosis of post-partum hemorrhage]. J Gynecol Obstet Biol Reprod (Paris) 2004;33:229-34. 23. Winter C, Macfarlane A, Deneux-Tharaux C, et al. Variations in policies for management of the third stage of labour and the immediate management of postpartum haemorrhage in Europe. Bjog 2007;114:845-54.

24. European consensus on prevention and management of postpartum haemorrhage, 2005. (Accessed at http://www.euphrates.inserm.fr/inserm/euphrates.nsf/AllDocumentsByUNID/95A14F46F31A 5246C125707400485AED?OpenDocument\&l=3.1.)

25. Donner A, Klar N. Design and analysis of cluster randomized trials in health research. London: Arnold; 2000.

26. Braunholtz DA, Edwards SJ, Lilford RJ. Are randomized clinical trials good for us (in the short term)? Evidence for a "trial effect". J Clin Epidemiol 2001;54:217-24.

27. Cheyne H, Hundley V, Dowding D, et al. Effects of algorithm for diagnosis of active labour: cluster randomised trial. Bmj 2008;337:a2396.

28. Buekens P, Alexander S, Boutsen M, Blondel B, Kaminski M, Reid M. Randomised controlled trial of routine cervical examinations in pregnancy. European Community Collaborative Study Group on Prenatal Screening. Lancet 1994;344:841-4.

29. Grant A, Elbourne D, Valentin L, Alexander S. Routine formal fetal movement counting and risk of antepartum late death in normally formed singletons. Lancet 1989;2:3459.

30. Althabe F, Buekens P, Bergel E, et al. A behavioral intervention to improve obstetrical care. N Engl J Med 2008;358:1929-40.

31. Prata N, Mbaruku G, Campbell M. Using the kanga to measure postpartum blood loss. Int J Gynaecol Obstet 2005;89:49-50. 


\section{Authors' statements}

\section{Competing interest statement}

All authors declare that the answer to the questions on your competing interest form are all No and therefore have nothing to declare.

\section{Copyright statement}

The Corresponding Author has the right to grant on behalf of all authors and does grant on behalf of all authors, an exclusive licence (or non exclusive for government employees) on a worldwide basis to the BMJ Publishing Group Ltd to permit this article (if accepted) to be published in BMJ editions and any other BMJPGL products and sublicences such use and exploit all subsidiary rights

\section{Contribution statements}

I declare that I participated in the design of the trial, the implementation of the trial in my country, the central monitoring of data collection, writing the statistical analysis plan, the cleaning and analysis of the data and the drafting and revision of the paper and that I have seen and approved the final version. I had full access to all the data in the study and had final responsibility for the decision to submit for publication. I have no conflicts of interest.

Wei-Hong Zhang

I declare that I participated in the design of the trial, the implementation of the trial in my country, the analysis of the data and the drafting and revision of the paper and that I have seen and approved the final version. I have no conflicts of interest.

Catherine Deneux-Tharaux

I declare that I participated in the design of the trial, the analysis of the data and the drafting and revision of the paper and that I have seen and approved the final version. I have no conflicts of interest.

Peter Brocklehurst

I declare that I participated in the conduct of the trial, writing the statistical analysis plan, the central monitoring of data collection and the revision of the draft paper and that I have seen and approved the final version. I have no conflicts of interest.

Edmund Juszczak

I declare that I participated in the design of the trial, the implementation of the trial in my country, the central monitoring of data collection and the revision of the draft paper and that I have seen and approved the final version. I have no conflicts of interest.

Matthew Joslin

I declare that I initiated the collaborative project, participated in the design of the trial, the implementation of the trial in my country, the central monitoring of data collection, the analysis of the data and the drafting and revision of the paper and that $I$ have seen and approved the final version. I have no conflicts of interest.

Sophie Alexander

I declare that I participated in the design of the trial, the implementation of the trial in my country and the revision of the draft paper and that I have seen and approved the final version. I have no conflicts of interest. 
I declare that I participated in the design of the trial, the implementation of the trial in my country and the revision of the draft paper and that I have seen and approved the final version. I have no conflicts of interest.

Istvan Berbik

I declare that I participated in the design of the trial, the implementation of the trial in my country and the revision of the draft paper and that I have seen and approved the final version. I have no conflicts of interest.

Marie-Hélène Bouvier-Colle

I declare that I participated in the design of the trial, the analysis of the data and the revision of the draft paper and that I have seen and approved the final version. I have no conflicts of interest.

Gérard Bréart

I declare that I participated in the design of the trial, the implementation of the trial in my country and the revision of the draft paper and that I have seen and approved the final version. I have no conflicts of interest.

Vicenç Cararach

I declare that I participated in the design of the trial, the implementation of the trial in my country and the revision of the draft paper and that I have seen and approved the final version. I have no conflicts of interest.

Risto Erkkola

I declare that I participated in the design of the trial, the implementation of the trial in my country and the revision of the draft paper and that I have seen and approved the final version. I have no conflicts of interest.

Mathias Klein

I declare that I participated in the design of the trial, the implementation of the trial in my country and the revision of the draft paper and that I have seen and approved the final version. I have no conflicts of interest.

Jens Langhoff-Roos

I declare that I participated in the design of the trial and the revision of the draft paper and that I have seen and approved the final version. I have no conflicts of interest.

Alison Macfarlane

I declare that I participated in the design of the trial, the implementation of the trial in my country and the revision of the draft paper and that I have seen and approved the final version. I have no conflicts of interest.

Walter Prendiville

I declare that I participated in the design of the trial, the implementation of the trial in my country and the revision of the draft paper and that I have seen and approved the final version. I have no conflicts of interest.

Jos van Roosmalen 
I declare that I participated in the design of the trial, the implementation of the trial in my country and the revision of the draft paper and that I have seen and approved the final version. I have no conflicts of interest.

Babill Stray-Pedersen

I declare that I participated in the design of the trial, the implementation of the trial in my country and the revision of the draft paper and that I have seen and approved the final version. I have no conflicts of interest.

Carolyn Troeger

I declare that I participated in the design of the trial and the revision of the draft paper and that I have seen and approved the final version. I have no conflicts of interest.

Clare Winter 